\title{
Diet and exercise interventions for pediatric cancer patients during therapy: tipping the scales for better outcomes
}

\author{
Keri L. Schadler ${ }^{1}$, Eugenie S. Kleinerman ${ }^{1}$ and Joya Chandra ${ }^{1}$
}

\begin{abstract}
Obesity at diagnosis is a negative prognostic indicator for several pediatric cancers including acute leukemia and bone tumors. Incidence of obesity in children has increased threefold over the past 2 decades, and causes for this include poor diet, excessive caloric intake, and lack of physical activity, which are collectively referred to as energy balance-related behaviors. Few energy balance interventions have been implemented in pediatric cancer patients during treatment, and here we will probe the rationale for pursuing such studies. The need to modify composition of calories consumed and to identify specific beneficial exercise regimens will be discussed, relative to weight reduction or management.
\end{abstract}

\section{BACKGROUND ON ENERGY BALANCE IN THE PEDIATRIC ONCOLOGY SETTING}

E nergy balance, the balance between energy intake (calorie consumption) and energy expenditure (physical activity $(\mathrm{PA})$ ), has gained interest in pediatric oncology in recent years. This is largely due to the obesity epidemic in healthy children as well as in children with cancer, and to the recognition in epidemiological and retrospective studies that obesity is a risk factor for several cancer types and for worse outcomes in some pediatric cancers. It is also due, in part, to increased interest from clinical care providers in improving the quality of life (QOL) for patients undergoing toxic anticancer treatments and to a growing understanding of how changes in energy balance affect the molecular biology of a tumor. Despite the relative ease of delivery of energy balance interventions, to date, there have been fewer than 30 clinical trials of PA interventions or diet/nutrition intervention studies in pediatric cancer patients during treatment regimens. Although systematic reviews for topics related to energy balance and cancer have been, and should continue to be, carried out, there are not enough energy balance interventions conducted in pediatric cancer patients during therapy to conduct a systematic review. The goal of this article is to prompt consideration of specific diet and exercise regimens that can be delivered during treatment to potentially optimize the efficacy of therapy and reduce the risk of treatment-related late effects. Many of these approaches may be ready for clinical implementation, such as moderate PA for prevention of functional decline; however, additional gaps in knowledge exist, such as determining the most appropriate calorie composition for patients with specific cancer types.

Over the past four decades, the use of combination chemotherapy treatment strategies has raised cure rates for children and adolescents with cancer to $80 \%$ (1). This improvement in success of treatment has been attributed to the effectiveness of combination chemotherapy, improved detection, classification, and diagnosis of the type of malignancy, and the ability to identify the presence of metastasis at the time of diagnosis (2). Efforts to design more specific and selective treatment modalities are still needed, and a majority of childhood cancer researchers are pursuing this goal in the hopes of further raising cure rates. However, less attention has been given to factors that may influence treatment outcomes that are amenable to behavior modification.

Energy balance, or the relationship between intake of food and PA, is one such factor that is within the control of cancer patients and one that can be altered through behavioral interventions (3). Much of the work investigating energy balance in the context of cancer treatment and survivorship has been focused on obesity, which can be caused by poor diet and sedentary lifestyles (4). Emerging data, primarily in adult cancers such as breast and prostate, highlight the relevance of obesity and energy balance to treatment outcomes and cure rates (5-7). Similar trends in pediatric oncology are developing. Recently, it was shown that obesity and the overall nutritional status of children with cancer contribute to treatment response and patient outcome (8-13). Pediatric patients who are obese or overweight have inferior survival rates, a higher rate of relapse, and are more resistant to therapy. Molecular explanations for this are postulated to be due to the altered pharmacokinetics of chemotherapy in overweight/obese patients and the inflammation-promoting interactions between adipocytes and cancer cells, which has been demonstrated in lab-based studies, but not in human studies as yet. Obesity rates in pediatric cancer patients have been tracked with age-matched controls globally and have

${ }^{1}$ Department of Pediatrics Research, Center for Energy Balance in Cancer Prevention and Survivorship, University of Texas MD Anderson Cancer Center, Houston, Texas. Correspondence: Joya Chandra (jchandra@mdanderson.org)

Received 25 January 2017; accepted 31 August 2017; advance online publication 25 October 2017. doi:10.1038/pr.2017.225 


\begin{tabular}{|c|c|c|}
\hline & Permissive diet & $\begin{array}{l}\text { - Pediatric ALL patients have a rapid increase in BMI following } \\
\text { diagnosis which persists into survivorship }{ }^{18} \\
\text { - Newly diagnosed pediatric cancer patients double their BMI and } \\
\text { fat mass by } 12 \text { months post diagonosis }{ }^{19} \\
\text { - Calorie consumption in newly diagonsed pediatric ALL patients is in } \\
\text { excess of daily recommended intake }{ }^{20}\end{array}$ \\
\hline & Prescribed diet & $\begin{array}{l}\text { - Low-carbohydrate (ketogenic or modified ketogenic) diet improves } \\
\text { efficacy of brain tumor therapy in mice }{ }^{39} \\
\text { - High-fat diet promotes tumor progression in multiple mouse } \\
\text { models for pancreatic and breast cancer }{ }^{34} \\
\text { - Breast cancer-brearing mice fed a high-sucrose diet have higher } \\
\text { rates of metastasis }{ }^{29}\end{array}$ \\
\hline & Unstructured/sedentary & $\begin{array}{l}\text { - Without exercise, cancer patients lose muscle strengh during } \\
\text { treatment }{ }^{50} \\
\text { - For pediatric cancer patients, each sedentary minute correlates } \\
\text { with reduced } \mathrm{VO}_{2 \text { peak }} 62\end{array}$ \\
\hline & Prescribed exercise & $\begin{array}{l}\text { - Exercise improves QOL in pediatric cancer patients undergoing } \\
\text { treatment }{ }^{56,57} \\
\text { - Resistance vs. aerobic exercise: unclear which is better and } \\
\text { benefits may be tumor type-dependent } 63,64 \\
\text { - Aerobic exercise improves chemotherapy efficacy in mice in } \\
\text { multiple tumor models }{ }^{63,67-69}\end{array}$ \\
\hline
\end{tabular}

Figure 1. Rationale for structured energy balance interventions in pediatric cancer patients. Permissive diets are associated with rapidly increased $\mathrm{BMI}$ and excess calorie consumption in pediatric cancer patients undergoing treatment. In contrast, prescribed diet in mouse models of brain, breast, and pancreatic cancer may improve therapy outcomes. Sedentary behaviors during cancer treatment are associated with loss of muscle strength and reduced cardiorespiratory endurance. In contrast, prescribed exercise enhances chemotherapy efficacy in mouse models and improves quality of life in patients.

increased almost three-fold in the past two decades (14). On the basis of these statistics, more newly diagnosed pediatric cancer patients are more likely to be obese than ever before.

However, a key question is whether simply changing diet habits and/or exercise habits vs. managing and reducing obesity will improve outcomes for these patients. Here we review the current knowledge of how obesity, nutritional status, and PA affect the treatment and outcome of children and adults with cancer and highlight studies that show the beneficial impact of implementing diet and PA regimens Figure 1.

\section{DIET-CALORIES VS. COMPOSITION}

Diet is certainly a component of the obesity problem and much data support the notion that food habits of pediatric cancer patients and survivors are suboptimal (15-17). This may be exacerbated with the use of steroid-containing chemotherapy regimens (18). Weight trajectories in childhood acute lymphoblastic leukemia (ALL) patients show that preschool children have a rapid rate of increase in overweight/ obesity following diagnosis and this frequently persists after therapy is complete (19). A meta-analysis of over 1,500 pediatric ALL patients in 16 studies concluded that BMI $z$ score increased early in treatment and in the maintenance phase and persisted beyond treatment into survivorship (20). These trends are seen in pediatric solid tumor and brain tumor patients as well, but the data on weight gain during treatment appear to be somewhat tumor-type-specific. A cohort of 133 newly diagnosed patients including brain and solid tumor patients showed increased BMI and fat mass within 3 months of diagnosis and doubled by 12 months post diagnosis (21). In contrast, weight loss occurred in 37\% of 468 patients with intermediate rhabdomyosarcoma and low baseline BMI correlated with borderline inferior survival (22). Collectively, these data indicate that weight management early during cancer treatment is an opportunity for interventions, and, to be successful, a nutritional program needs to be integrated into the clinical care model and must be sustained throughout treatment (23). However, research remains to be carried out to define precisely what "obesity management" or "good nutrition" means for these patients. Calorie reduction with no focus on the calorie composition or which foods the calories are coming from may not be adequate to improve outcomes. For example, it is unclear whether reduced intake of sugars, carbohydrates, or fat vs. increased protein intake or increased consumption of fruits and vegetables will be beneficial.

A recent study examining diet in 640 newly diagnosed pediatric acute lymphocytic leukemia patients found that caloric consumption was in excess of daily recommended intake, and that consumption of vitamin $\mathrm{E}$ and $\mathrm{D}$ was below recommended daily allowance (24). These nutritional deficiencies appear to extend beyond treatment, as in pediatric cancer survivors spanning diagnoses and age groups, fruit and vegetable consumption is low (25). ALL survivors consumed less vitamin $\mathrm{D}$, calcium, fiber, and potassium than the recommended guidelines, but exceeded the saturated fat and sodium-intake guidelines (17). In an effort to improve bone mineral density in childhood ALL survivors, which is compromised because of corticosteroids and methotrexate treatment regimens, a randomized supplementation trial using calcium and vitamin D or placebo was carried out in 


\section{Review | schadler et al.}

275 participants over a 2-year timespan (26). Although bone density did not improve in the intervention group, the dosage of Vitamin D and the timing of supplementation relative to chemotherapy exposure are variables that require consideration before drawing negative conclusions regarding the potential benefits of supplementation. In addition, rather than using supplements, whole foods that are nutrient-dense across food groups as per the 2015-2020 Dietary Guidelines for Americans could be employed during delivery of steroid or methotrexate-intensive treatment regimens for pediatric cancer patients.

The obesity problem has been attributed in part to increased consumption of sugar and fat (27). Whether intake of sugar and fat contributes to poor outcomes in cancer patients independent of obesity is unclear. For example, epidemiological data show that sugar intake correlates with increased cancer incidence, and mouse models for breast and liver cancer indicate that sucrose-rich diets promote tumor progression and metastasis in normal-weight non-obese mice (28-32). Molecular explanations for the impact of sugar on tumor progression have been put forth from patient data and mouse models. Specific metabolites associated with the lipoxygenase pathway have also been correlated with sugar consumption. The lipoxygenase pathway, as well as activation of the IGF pathway, is linked to increased levels of intracellular and extracellular reactive oxygen species. Similarly, high levels of protein and fat consumption generate oxidative stress, which can promote cancer progression (33). A series of studies have documented that elevated serum levels of IGFBP2 are predictive of increased risk of recurrence in pediatric leukemia patients (34-36). Little is known about dietary influences on IGFBP2; however, a cross-sectional analysis of 4,731 men and women enrolled in the European Prospective Investigation into Cancer and Nutrition study showed an association between dairy protein consumption and circulating IGFBP2 concentrations (37). These data suggest that for patients on therapy, high-sugar diets should be avoided and protein and fat consumption needs to be monitored. In stark contrast to this concept, recent data show that obese melanoma patients or those with a high BMI had a better response to kinase inhibitor therapy (38) and that in mouse models, a ketogenic, high-fat, low-carbohydrate diet inhibited the growth and progression of glioblastoma (39). Therefore, tailoring of diet recommendations to tumor type and therapy type is critical and suggests that perhaps the calorie composition rather than the total calories or the patient's BMI should be the focus of behavioral interventions aimed at changing diet.

Mouse modeling of energy balance interventions using pediatric models will yield data necessary to optimize a diet regimen with the potential to improve treatment outcomes, but delivery in patients poses additional challenges. Few dietary interventions have been implemented in pediatric cancer patients and survivors, and emphasis has primarily been on healthy lifestyles and nutrition education rather than on the use of a specific or prescribed diet (40-42).
Surprisingly, the strongest data in this field have come from ketogenic diets (43). Long used for epilepsy patients, ketogenic diet improves therapy efficacy for brain tumors in mouse models (39). Only one small case study of two pediatric patients with astrocytoma tumors has been published to date and demonstrated decreased glucose uptake and improved mood in one patient using ketogenic diets $(44,45)$. However, the feasibility of delivery of these diets to children is questionable, as is the drastic caloric restriction diets that show increased efficacy in mouse models for prostate, breast, and ovarian cancers (46). Limited data in adult glioma patients (47) indicate the safety and feasibility of the ketogenic diet, caloric restriction, as well as minor modifications of these regimens and encourages its use as an adjunctive therapy. As described above, high-sugar and high-fat diets are being documented to have a negative impact on treatment outcomes, whereas enrichment for other macro- or micronutrients could have beneficial effects. In children, a major determinant of diet interventions involves parental practices. Although short-term diet interventions in a hospital setting could be controlled through collaboration between physicians and dieticians, diet change in an outpatient setting requires education and perhaps even interventions designed for parents (48). These types of interventions must be matched culturally, socioeconomically, and with attention to time required for food preparation and unique palates of children with cancer, which may be changed because of side effects of treatment such as nausea and altered senses of taste and smell. Despite these challenges, a recent systematic review found that adherence to lifestyle interventions improves when a parental component is included (49).

\section{EXERCISE-FEASIBILITY AND TIMING}

Although diet modification can have a role in controlling obesity and BMI, there can be adverse effects of diet-induced weight loss on muscle strength (50). There can be a significant loss of fat-free mass depending on the diet modification and the calorie reduction. Strategies such as weight training or aerobic exercise can combat the loss of muscle mass and strength (50). PA can also have a role in controlling BMI and obesity as energy balance is defined as calories consumedenergy expended. Therefore, it is important for a nutritional program for cancer patients to also include a PA component to increase the number of calories burned and prevent loss of muscle mass. Exercise has been shown to be both feasible and safe in Pediatric cancer patients undergoing chemotherapy (51). Exercise has also been shown to improve the QOL of cancer patients and reduce the risk of cardiovascular disease in cancer survivors $(52,53)$. However, there is still hesitancy by clinicians to use PA in the cancer-care setting, largely because of uncertainties about how to appropriately prescribe it. First, it is unclear when PA is most beneficial. Most epidemiological studies have been used to support exercise as part of a healthy lifestyle for cancer prevention, whereas most clinical studies have used PA/exercise in the survivorship setting as part of obesity management. One growing field of 
research focuses on the use of exercise during active therapy for a cancer patient-the topic of this review. In addition, it is unclear whether exercise quantity, quality, or both is most important. Owing to the large variability in experimental design in published studies in terms of type of exercise, frequency, and duration of the intervention program, identifying the "best" exercise program is challenging. Finally, the relationship between obesity and exercise efficacy is not well defined. It remains unknown whether exercise can have beneficial effects even in the absence of weight control.

Exercise or PA during cancer treatment can be used for three main purposes: to improve the QOL of a cancer patient (psychosocial benefits), to prevent decline of physical functioning and secondary medical complications (obesity prevention and atrophy prevention), and to improve the efficacy of chemotherapy. The data for each of these broad categories are described below, with an emphasis on data specific to pediatric patients. Studies in adults have unequivocally demonstrated improved QOL for cancer patients who are physically active, reporting decreased fatigue, increased mobility, and decreased depression $(54,55)$. The pediatric literature is much less robust, as fewer studies using PA/ exercise have been performed in pediatric cancer patients. Those that have been performed support a positive effect of PA on QOL, with varying levels of significance, and have been well reviewed $(56,57)$. Importantly, a reduction in pain and perceived procedure anxiety was achieved by a combined PA and psychosocial intervention for patients within 1 year of therapy (58). Similarly, a 6-month group exercise program for outpatients of mixed diagnoses was shown to significantly improve overall motor performance, activity level, and emotional well-being (59). Although improved QOL is an accepted benefit of PA/exercise for cancer patients, other benefits are also supported by research. The six randomized, prospective clinical trials that have been performed in pediatric patients undergoing treatment for ALL have recently been summarized (60). In all studies, cardiorespiratory fitness was significantly improved by exercise, as measured by the 9min walk test, despite heterogeneity in exercise programs. Similarly, in a small study of 27 ALL participants receiving maintenance therapy in their first remission, a home-based exercise program was shown to achieve significant improvement in a 6-min walk test performance for $75 \%$ of participants. The mean BMI change for participants in this study was a decrease of $4.2 \%$, indicating that a PA plan performed while on chemotherapy can reduce obesity (61). It is unclear, however, whether a reduction in obesity is necessary to achieve the numerous other benefits associated with PA. In a study of 60 children with any type of malignancy, either actively on therapy or within a year of completion, PA was again demonstrated to be significantly correlated to cardiorespiratory fitness. This randomized controlled trial consisted of a 12-week exercise and psychosocial program. Each additional activity count per minute correlated with an increase of $0.05 \mathrm{ml} / \mathrm{kg} / \mathrm{min}$ in $\mathrm{VO}_{2 \text { peak }}$. Conversely, each sedentary minute reduced $\mathrm{VO}_{2 \text { peak }}$ by
$0.06 \mathrm{ml} / \mathrm{kg} / \mathrm{min}$. This study also confirmed that pediatric cancer patients are less physically fit than their age-matched healthy counterparts, with significantly lower $\mathrm{VO}_{2 \text { peak }}$ (ref. 62). These studies demonstrate that exercise for pediatric cancer patients is feasible. However, the most effective type of exercise (aerobic vs. resistance) has not been compared in pediatric populations.

In a recent study comparing aerobic exercise (motorized wheel running) to resistance training (weighted ladder climbing) as a means of preventing cancer-associated cachexia using the C26 mouse colon cancer model, Khamoui et al. demonstrated that aerobic exercise preserved relative grip strength, sensorimotor function, and muscle mass whereas resistance training did not. In fact, $25 \%$ of mice undergoing resistance training became moribund before the end of the study, whereas none of the mice performing aerobic exercise became moribund. However, all mice lost total mass, suggesting that these exercise regimens alone are not sufficient to protect against cachexia (63). In stark contrast to the findings in mice, a recent study in breast cancer patients undergoing chemotherapy compared three times weekly aerobic exercise with three times weekly resistance training. In this study, resistance exercise training improved sarcopenia and dynapenia significantly more than aerobic exercise (64). These nearly opposite findings accentuate the need for more controlled clinical trials comparing exercise types, intensities, and frequencies, as each of these variables is likely to cause different outcomes.

Exercise during therapy is feasible and safe for pediatric cancer patients, and it improves physical fitness (56). PA or exercise may also improve therapeutic efficacy. To date, no clinical trials in humans have examined the effect of PA on treatment efficacy. Animal studies using tumor-bearing mice inform our understanding of the usefulness of exercise as an adjuvant to chemotherapy. Unfortunately, the majority of animal studies have been conducted using models of adult cancers; however, these may have relevance to pediatric oncology. The effect of exercise on tumor growth in the absence of other therapies appears to be tumor-typedependent. For example, wheel running inhibited growth of A549 lung tumors and ET1 mammary tumors in mice, but had no effect on growth of orthotopic prostate tumors (65-67). Wheel running and treadmill exercise both promoted the growth of tumors in $p 53^{+/-} ; M M T V-W n t-1$ transgenic mice (68). Although exercise alone appears to affect different tumor types differently, exercise has consistently been demonstrated to improve chemotherapy efficacy. Five days per week of high-intensity treadmill running alone had no effect on tumor growth in mice bearing orthotopic glioblastoma tumors, but increased survival and decreased tumor growth when combined with temozolomide (TMZ) compared with TMZ alone (69). Similarly, treadmill running at a more moderate intensity increased the efficacy of gemcitabine against pancreatic ductal adenocarcinoma and of doxorubicin against melanoma in mice (70). Voluntary wheel running also significantly increased the efficacy of 
chemotherapy in multiple models. Wheel running significantly increased the antitumor effect of cyclophosphamide against mammary tumors and of celecoxib against prostate tumors in mice $(65,71)$. The heterogeneity in exercise prescriptions between these studies suggests that there may be a wide range of aerobic exercise that is beneficial to improve outcome during chemotherapy. No weight loss due to exercise was reported for mice in any of these studies, indicating that exercise may be efficacious as a therapeutic adjuvant regardless of changes in weight. However, it must be noted that these studies did not use obese mice, and therefore the importance of reducing obesity cannot be evaluated with the current data.

\section{CONCLUSIONS}

In summary, the studies cited above suggest that diet quality and/or specific exercise parameters, which can contribute to obesity prevention and management, are important factors for improving outcomes for pediatric cancer patients. These studies provide impetus for investigation of focused nutritional and PA programs that manage or prevent obesity and promote healthy lifestyles in the context of the treatment of pediatric cancer patients. Patients with genetic predisposition to obesity will likely require different approaches. For example, mutations in the leptin gene (which encodes a hormone synthesized primarily by adipocytes that acts on its receptor in the hypothalamus and conveys feelings of satiety) in mice and in humans are associated with mild-to-severe forms of obesity (72,73). Experiments using mice bearing mutations in the leptin-melanocortin pathway showed that caloric restriction and exercise were non-identical to strainmatched lean mice in terms of body fat composition, gene expression, and blood lipid levels (74). These data suggest that screening patients for monogenic or polygenic obesity could be useful in determining whether energy balance interventions alone will exert beneficial effects or whether pharmacological approaches would be required to target these pathways.

Collectively, answering questions raised in this review will require concerted efforts of clinicians, researchers, and behavioral scientists, but has potential to improve chemotherapy efficacy without raising the dose and accompanying toxicities/late effects, and represents a relatively inexpensive supportive care intervention. Cooperative groups and consortiums are an ideal setting for carrying out these trials; however, it would require that a consensus be reached regarding exactly what exercise and/or diet modification would be implemented during standard of care regimens for the most common pediatric cancers in order to determine whether improved outcomes are seen. With regard to exercise this may be easier, given the many reports of feasibility of moderate exercise regimens in pediatric cancer patients and the proliferation of activity trackers, which can be used to monitor compliance to prescribed PA. For diet, challenges to implementation of a specific nutritional regimen during treatment include a lack of consensus regarding how to assess adequate nutrition, and a lack of measures for diet compliance. Self-report of diet is inherently flawed, and further complicated in pediatric populations, and reliable biomarkers of diet modification (such as inflammation or oxidative stress-related biomarkers) are confounded by factors related to cancer type, modality of treatment, and patient-topatient variability. These challenges provide impetus for future work in this area to clarify exactly how energy balance can be leveraged in the treatment setting for pediatric oncology patients.

\section{ACKNOWLEDGMENTS}

These authors are grateful for support from the following sources: P30 CA16672; Center for Energy Balance in Cancer Prevention and Survivorship, Multidisciplinary Research Grant Program, and the Archer Family Foundation.

Disclosure: The authors declare no conflict of interest.

\section{REFERENCES}

1. Smith MA, Seibel NL, Altekruse SF, et al. Outcomes for children and adolescents with cancer: challenges for the twenty-first century. J Clin Oncol 2010;28:2625-34.

2. Reaman GH. Pediatric cancer research from past successes through collaboration to future transdisciplinary research. J Pediatr Oncol Nurs 2004;21:123-7.

3. Ligibel JA, Alfano CM, Hershman D, et al. Recommendations for obesity clinical trials in cancer survivors: American Society of Clinical Oncology Statement. J Clin Oncol 2015;33:3961-7.

4. Klil-Drori AJ, Azoulay L, Pollak MN. Cancer, obesity, diabetes, and antidiabetic drugs: is the fog clearing? Nat Rev Clin Oncol 2016;14:85-99.

5. Wang LS, Murphy CT, Ruth K, et al. Impact of obesity on outcomes after definitive dose-escalated intensity-modulated radiotherapy for localized prostate cancer. Cancer 2015;121:3010-7.

6. Matthews SB, Thompson HJ. the obesity-breast cancer conundrum: an analysis of the issues. Int J Mol Sci 2016;17:989-1008.

7. Laurent V, Guerard A, Mazerolles C, et al. Periprostatic adipocytes act as a driving force for prostate cancer progression in obesity. Nat Commun 2016;7:10230.

8. Orgel E, Tucci J, Alhushki W, et al. Obesity is associated with residual leukemia following induction therapy for childhood B-precursor acute lymphoblastic leukemia. Blood 2014;124:3932-8.

9. Sala A, Rossi E, Antillon F, et al. Nutritional status at diagnosis is related to clinical outcomes in children and adolescents with cancer: a perspective from Central America. Eur J Cancer 2012;48:243-52.

10. Orgel E, Sposto R, Malvar J, et al. Impact on survival and toxicity by duration of weight extremes during treatment for pediatric acute lymphoblastic leukemia: a report from the Children's Oncology Group. J Clin Oncol 2014;32:1331-7.

11. Lange BJ, Gerbing RB, Feusner J, et al. Mortality in overweight and underweight children with acute myeloid leukemia. JAMA 2005;293: 203-11.

12. Butturini AM, Dorey FJ, Lange BJ, et al. Obesity and outcome in pediatric acute lymphoblastic leukemia. J Clin Oncol 2007;25:2063-9.

13. Goldstein G, Shemesh E, Frenkel T, Jacobson JM, Toren A. Abnormal body mass index at diagnosis in patients with Ewing sarcoma is associated with inferior tumor necrosis. Pediatr Blood Cancer 2015;62:1892-6.

14. Rogers PC, Meacham LR, Oeffinger KC, Henry DW, Lange BJ. Obesity in pediatric oncology. Pediatr Blood Cancer 2005;45:881-91.

15. Badr H, Chandra J, Paxton RJ, et al. Health-related quality of life, lifestyle behaviors, and intervention preferences of survivors of childhood cancer. J Cancer Surviv 2013;7:523-34. 
16. Cohen J, Wakefield CE, Fleming CA, Gawthorne R, Tapsell LC, Cohn RJ. Dietary intake after treatment in child cancer survivors. Pediatr Blood Cancer 2012;58:752-7.

17. Zhang FF, Saltzman E, Kelly MJ, et al. Comparison of childhood cancer survivors' nutritional intake with US dietary guidelines. Pediatr Blood Cancer 2015;62:1461-7.

18. Zhang FF, Rodday AM, Kelly MJ, et al. Predictors of being overweight or obese in survivors of pediatric acute lymphoblastic leukemia (ALL). Pediatr Blood Cancer 2014;61:1263-9.

19. Winkler MR, Hockenberry MJ, McCarthy KS, Silva SG. Trajectories of obesity and overweight rates among survivors of childhood acute lymphoblastic leukemia. Oncol Nurs Forum 2015;42:E287-93.

20. Zhang FF, Liu S, Chung M, Kelly MJ. Growth patterns during and after treatment in patients with pediatric ALL: a meta-analysis. Pediatr Blood Cancer 2015;62:1452-60.

21. Brinksma A, Roodbol PF, Sulkers E, et al. Changes in nutritional status in childhood cancer patients: a prospective cohort study. Clin Nutr 2015;34: 66-73.

22. Burke ME, Lyden ER, Meza JL, et al. Does body mass index at diagnosis or weight change during therapy predict toxicity or survival in intermediate risk rhabdomyosarcoma? A report from the Children's Oncology Group Soft Tissue Sarcoma Committee. Pediatr Blood Cancer 2013;60:748-53.

23. Ladas EJ, Arora B, Howard SC, Rogers PC, Mosby TT, Barr RD. A framework for adapted nutritional therapy for children with cancer in low- and middle-income countries: a report from the SIOP PODC Nutrition Working Group. Pediatr Blood Cancer 2016;63:1339-48.

24. Ladas EJ, Orjuela M, Stevenson K, et al. Dietary intake and childhood leukemia: the Diet and Acute Lymphoblastic Leukemia Treatment (DALLT) cohort study. Nutrition 2016;32:1103-9 e1.

25. Warner EL, Nam GE, Zhang Y, et al. Health behaviors, quality of life, and psychosocial health among survivors of adolescent and young adult cancers. J Cancer Surviv 2016;10:280-90.

26. Kaste SC, Qi A, Smith K, et al. Calcium and cholecalciferol supplementation provides no added benefit to nutritional counseling to improve bone mineral density in survivors of childhood acute lymphoblastic leukemia (ALL). Pediatr Blood Cancer 2014;61:885-93.

27. Norat T, Scoccianti C, Boutron-Ruault MC, et al. European code against cancer 4th edition: diet and cancer. Cancer Epidemiol 2015;39 (Suppl 1): S56-66.

28. Melkonian SC, Daniel CR, Ye Y, Pierzynski JA, Roth JA, Wu X. Glycemic index, glycemic load, and lung cancer risk in non-hispanic whites. Cancer Epidemiol Biomarkers Prev 2016;25:532-9.

29. Jiang $Y$, Pan $Y$, Rhea PR, et al. A sucrose-enriched diet promotes tumorigenesis in mammary gland in part through the 12-lipoxygenase pathway. Cancer Res 2016;76:24-9.

30. Healy ME, Lahiri S, Hargett SR, et al. Dietary sugar intake increases liver tumor incidence in female mice. Sci Rep 2016;6:22292.

31. Qin B, Moorman PG, Alberg AJ, et al. Dietary carbohydrate intake, glycaemic load, glycaemic index and ovarian cancer risk in AfricanAmerican women. Br J Nutr 2016;115:694-702.

32. Sulaiman S, Shahril MR, Wafa SW, Shaharudin SH, Hussin SN. Dietary carbohydrate, fiber and sugar and risk of breast cancer according to menopausal status in Malaysia. Asian Pac J Cancer Prev 2014;15:5959-64.

33. Martinez-Useros J, Garcia-Foncillas J. Obesity and colorectal cancer: molecular features of adipose tissue. J Transl Med 2016;14:21.

34. Kitszel A, Krawczuk-Rybak M. Are elevated serum levels of IGFBP-2 after intensive chemotherapy of childhood acute lymphoblastic leukemia a risk factor of relapse? Adv Med Sci 2007;52:147-53.

35. Vorwerk $\mathrm{P}$, Mohnike $\mathrm{K}$, Wex $\mathrm{H}$, et al. Insulin-like growth factor binding protein-2 at diagnosis of childhood acute lymphoblastic leukemia and the prediction of relapse risk. J Clin Endocrinol Metab 2005;90: 3022-7.

36. Dawczynski K, Kauf E, Schlenvoigt D, Gruhn B, Fuchs D, Zintl F. Elevated serum insulin-like growth factor binding protein-2 is associated with a high relapse risk after hematopoietic stem cell transplantation in childhood AML. Bone Marrow Transplant 2006;37:589-94.
37. Crowe FL, Key TJ, Allen NE, et al. The association between diet and serum concentrations of IGF-I, IGFBP-1, IGFBP-2, and IGFBP-3 in the European Prospective Investigation into Cancer and Nutrition. Cancer Epidemiol Biomarkers Prev 2009;18:1333-40.

38. McQuade J. The impact of obesity on outcomes in metastatic melanoma (MM) patients (pts) treated with dabrafenib and trametinib. J Clin Oncol 2016;34:9566.

39. Klement RJ, Champ CE, Otto C, Kammerer U. Anti-tumor effects of ketogenic diets in mice: a meta-analysis. PLoS ONE 2016;11:e0155050.

40. Selwood K, Ward E, Gibson F. Assessment and management of nutritional challenges in children's cancer care: a survey of current practice in the United Kingdom. Eur J Oncol Nurs 2010;14:439-6.

41. Wu YP, Yi J, McClellan J, et al. Barriers and facilitators of healthy diet and exercise among adolescent and young adult cancer survivors: implications for behavioral interventions. J Adolesc Young Adult Oncol 2015;4:184-91.

42. Moyer-Mileur LJ, Ransdell L, Bruggers CS. Fitness of children with standard-risk acute lymphoblastic leukemia during maintenance therapy: response to a home-based exercise and nutrition program. J Pediatr Hematol Oncol 2009;31:259-66.

43. Allen BG, Bhatia SK, Anderson CM, et al. Ketogenic diets as an adjuvant cancer therapy: History and potential mechanism. Redox Biol 2014;2:963-70.

44. Nebeling LC, Lerner E. Implementing a ketogenic diet based on mediumchain triglyceride oil in pediatric patients with cancer. J Am Diet Assoc 1995;95:693-7.

45. Nebeling LC, Miraldi F, Shurin SB, Lerner E. Effects of a ketogenic diet on tumor metabolism and nutritional status in pediatric oncology patients: two case reports. J Am Coll Nutr 1995;14:202-8.

46. Brandhorst S, Longo VD. Fasting and caloric restriction in cancer prevention and treatment. Recent Results Cancer Res 2016;207:241-66.

47. Schwartz K, Chang HT, Nikolai M, et al. Treatment of glioma patients with ketogenic diets: report of two cases treated with an IRB-approved energy-restricted ketogenic diet protocol and review of the literature. Cancer Metab 2015;3:3.

48. Stern M, Ewing L, Davila E, Thompson AL, Hale G, Mazzeo S. Design and rationale for NOURISH-T: a randomized control trial targeting parents of overweight children off cancer treatment. Contemp Clin Trials 2015;41:227-37.

49. Raber M, Swartz MC, Santa Maria D, et al. Parental involvement in exercise and diet interventions for childhood cancer survivors: a systematic review. Pediatr Res 2016;80:338-46.

50. Zibellini J, Seimon RV, Lee CM, Gibson AA, Hsu MS, Sainsbury A. Effect of diet-induced weight loss on muscle strength in adults with overweight or obesity - a systematic review and meta-analysis of clinical trials. Obes Rev 2016;17:647-3.

51. Huang T-T. Exercise interventions in children with cancer: a review. Int J Pediatrics 2011;2011:461512.

52. Mishra SI, Scherer RW, Snyder C, Geigle P, Gotay C. Are exercise programs effective for improving health-related quality of life among cancer survivors? A systematic review and meta-analysis. Oncol Nurs Forum 2014;41:E326-42.

53. Kirkham AA, Davis MK.. exercise prevention of cardiovascular disease in breast cancer survivors. J Oncol 2015;2015:917606.

54. Lucia A, Earnest C, Perez M. Cancer-related fatigue: can exercise physiology assist oncologists? Lancet Oncol 2003;4:616-25.

55. Buffart L, Ros W, Chinapaw M, Brug J, Knol D, Korstjens I. Mediators of physical exercise for improvement in cancer survivors' quality of life. Psychooncology 2014;23:330-8.

56. Baumann FT, Bloch W, Beulertz J. Clinical exercise interventions in pediatric oncology: a systematic review. Pediatr Res 2013;74:366-74.

57. Tseng-Tien H, Kirsten KN. "Exercise Interventions in Children with Cancer: A Review," International Journal of Pediatrics, vol. 2011, Article ID 461512, 11 pages, 2011. doi:10.1155/2011/461512.

58. van Dijk-Lokkart EM, Braam KI, van Dulmen-den Broeder E, et al. Effects of a combined physical and psychosocial intervention program for childhood cancer patients on quality of life and psychosocial functioning: results of the QLIM randomized clinical trial. Psycho-Oncology 2016;25: 815-22. 


\section{Review | Schadler et al.}

59. Beulertz J, Prokop A, Rustler V, Bloch W, Felsch M, Baumann FT. Effects of a 6-month, group-based, therapeutic exercise program for childhood cancer outpatients on motor performance level of activity, and quality of life. Pediatr Blood Cancer 2016;63:127-32.

60. Braam KI, van der Torre P, Takken T, Veening MA, van Dulmen-den Broeder E, Kaspers GJL. Physical exercise training interventions for children and young adults during and after treatment for childhood cancer. Cochrane Database Syst Rev 2016;3:CD008796.

61. Esbenshade AJ, Friedman DL, Smith WA, et al. Feasibility and initial effectiveness of home exercise during maintenance therapy for childhood acute lymphoblastic leukemia. Pediatr Phys Ther 2014;26:301-7.

62. Braam KI, van Dijk-Lokkart EM, Kaspers GJL, et al. Cardiorespiratory fitness and physical activity in children with cancer. Support Care Cancer 2016;24:2259-68.

63. Khamoui AV, Park B-S, Kim D-H, et al. Aerobic and resistance training dependent skeletal muscle plasticity in the colon-26 murine model of cancer cachexia. Metabolism 2016;65:685-98.

64. Adams SC, Segal RJ, McKenzie DC, et al. Impact of resistance and aerobic exercise on sarcopenia and dynapenia in breast cancer patients receiving adjuvant chemotherapy: a multicenter randomized controlled trial. Breast Cancer Res Treat 2016;158:497-507.

65. Betof AS, Lascola CD, Weitzel DH, et al. Modulation of murine breast tumor vascularity, hypoxia, and chemotherapeutic response by exercise. J Natl Cancer Inst 2015: 107.

66. Jones LW, Antonelli J, Masko EM, et al. Exercise modulation of the hosttumor interaction in an orthotopic model of murine prostate cancer. J Appl Physiol 2012;113:263-72.
67. Higgins KA, Park D, Lee GY, Curran WJ, Deng X. Exercise-induced lung cancer regression: mechanistic findings from a mouse model. Cancer 2014;120:3302-10.

68. Colbert LH, Westerlind KC, Perkins SN, et al. Exercise effects on tumorigenesis in a p53-deficient mouse model of breast cancer. Med Sci Sports Exerc 2009;41:1597-605.

69. Lemke D, Pledl H-W, Zorn M, et al. Slowing down glioblastoma progression in mice by running or the anti-malarial drug dihydroartemisinin? Induction of oxidative stress in murine glioblastoma therapy. Oncotarget 2016;7:56713-25.

70. Schadler KL, Thomas NJ, Galie PA, et al. Tumor vessel normalization after aerobic exercise enhances chemotherapeutic efficacy. Oncotarget 2016;7:65429-40.

71. Zheng XI, Cui X-X, Gao ZHI, et al. Inhibitory effect of dietary atorvastatin and celecoxib together with voluntary running wheel exercise on the progression of androgen-dependent LNCaP prostate tumors to androgen independence. Exp Ther Med 2011;2:221-8.

72. Fischer-Posovszky P, von Schnurbein J, Moepps B, et al. A new missense mutation in the leptin gene causes mild obesity and hypogonadism without affecting $\mathrm{T}$ cell responsiveness. J Clin Endocrinol Metab 2010;95: 2836-40.

73. Montague CT, Farooqi IS, Whitehead JP, et al. Congenital leptin deficiency is associated with severe early-onset obesity in humans. Nature 1997;387:903-8.

74. Chiu S, Fisler JS, Espinal GM, Havel PJ, Stern JS, Warden CH. The yellow agouti mutation alters some but not all responses to diet and exercise. Obes Res 2004;12:1243-55. 